\title{
Parametric study on the hygrothermal performance of timber frame walls with external airtightness membranes in a temperate maritime climate
}

\author{
Valentina Marincioni ${ }^{1,2^{*}}$, Federico Lorenzetti ${ }^{2}$, and Hector Altamirano-Medina ${ }^{1,2}$ \\ ${ }^{1}$ UCL Institute for Environmental Design and Engineering, 14 Upper Woburn Place, London, \\ WC1H 0NN, United Kingdom \\ ${ }^{2}$ UK Centre for Moisture in Buildings (UKCMB), UCL Here East, 8-9 East Bay Lane, Queen \\ Elizabeth Olympic Park, E15 2GW, UK
}

\begin{abstract}
In recent years, external airtightness membranes have become an option for timber frame wall systems, as they allow high levels of airand wind-tightness with an easy installation and provide rainwater protection during construction. This opens up the option of removing the internal air and vapour control layer. However, the hygrothermal risks associated to this option could be higher than in conventional construction, because vapour transfer can occur from the indoor environment into the timber frame wall not just via diffusion but also advection. This can lead to moisture accumulation and mould growth risk within the wall structure. This paper presents a parametric study that aims at identifying the moisture risk when external airtightness membranes are installed on a timber frame wall in a temperate maritime climate. The parametric study considered the two-dimensional heat, air and moisture transfer within a timber frame wall. Parameters having higher influence on moisture risk were identified and should be considered when designing robust wall systems.
\end{abstract}

\section{Introduction}

The aim of installing airtightness membranes behind the plasterboard is to reduce the air flow between the indoor environment and the building fabric and the outflow of water vapour present in air. Most airtightness membranes can also help reducing vapour diffusion through the wall; these are called Air and Vapour Control Layers (AVCL).

Concerning moisture transfer, it has been found that flow of bulk air has a higher impact on moisture-related damage [1] than vapour diffusion flow, as it carries far more water vapour than diffusion [2]. The extent of air flow control depends on the air permeability of the airtightness membrane, but also - and more importantly - on the level/quality of sealing between membranes. The extent of vapour diffusion control mainly depends on the vapour diffusion resistance of the membrane.

The AVCL is usually located behind the plasterboard. Yet, external airtightness membranes are recently being offered for framed walls as an easy-to-build way of ensuring greater airtightness (as there is no breaching of the membrane due to services), as well as providing wind-tightness and reducing construction moisture.

\footnotetext{
* Corresponding author: v.marincioni@ucl.ac.uk
} 
Having an external airtightness membrane opens up the option of removing the internal AVCL. However, this could lead to increased moisture risks, allowing greater air movement from the indoor environment towards the lightweight wall structure. Leakages in airtightness layers between plasterboard and insulation had been found to lead to unwanted exfiltration and higher interstitial condensation; also, air convection within air permeable insulation has been found to affect the moisture redistribution within lightweight walls.

The common build-up of a timber frame wall in the United Kingdom considers an Oriented Strand Board (OSB) as sheathing board, located to the outside of the insulation. As the OSB has a relatively high vapour resistance and is at low temperature, vapour condensation can occur at its internal surface if any vapour enters the wall. This paper presents an analysis of the risks associated to moisture accumulation at the internal surface of OSB, with a focus on the application of external airtightness membranes.

\section{Method}

A parametric study was performed to identify the moisture risk associated to material properties, when external airtightness membranes are installed. The parameters studied were vapour resistance of internal AVCL and presence of air gaps, air permeability, vapour permeability and thickness of mineral wool, and vapour permeability and thermal conductivity of OSB.

\subsection{Simulation software}

DELPHIN 6.0 simulation software was used for two-dimensional Heat, Air and Moisture transfer simulations, because it accounts for gravity driven (buoyancy) air transfer [3]. The moisture transfer mechanisms evaluated were air pressure difference and stack-induced pressures for airflow, vapour diffusion and liquid redistribution.

\subsection{Input data}

The base case studied is represented by an insulated framework with (outside to inside) an external airtightness membrane, $12 \mathrm{~mm}$ of OSB as sheathing board, $100 \mathrm{~mm}$ of mineral wool as insulation, an AVCL, and $12.5 \mathrm{~mm}$ of standard gypsum plasterboard as internal finish (as shown in Table 1). Air gaps on the internal finish and AVCL were implemented in the simulation by adding $5 \mathrm{~cm}$ at the top and bottom of the wall. It was assumed that the external finish is made of timber cladding, behind which there is a well-ventilated layer. For simulation purposes, cladding was not included in the cross section; instead, it was represented by a convective heat transfer coefficient that was lower than the default coefficient for external surfaces, according to BS EN ISO 6946 [4]. Boundary conditions are presented in Table 2 .

Table 1. Material data for parametric study.

\begin{tabular}{|c|c|c|c|c|}
\hline $\begin{array}{l}\text { Element } \\
\text { (outside to inside) }\end{array}$ & $\begin{array}{l}\text { Material for } \\
\text { simulation }\end{array}$ & Key parameters & Base case & $\begin{array}{l}\text { Values for } \\
\text { parametric study }\end{array}$ \\
\hline \multirow[t]{4}{*}{ Airtightness membrane } & Wraptite & $\mathrm{S}_{\mathrm{d}}$ & $0.039 \mathrm{~m}$ & \\
\hline & & $\begin{array}{l}\text { Design air } \\
\text { permeability }\end{array}$ & $1 \mathrm{E}-7 \mathrm{~s}$ & \\
\hline & & Leakage & None & \\
\hline & & Thickness & $0.65 \mathrm{~mm}$ & \\
\hline \multirow[t]{3}{*}{ Sheathing board } & OSB & $\mu$ & 280 & $165,280,400,600,800$ \\
\hline & & $\lambda$ & $0.3 \mathrm{~W} /(\mathrm{mK})$ & $0.03,0.3,0.6,1$ \\
\hline & & Thickness & $12 \mathrm{~mm}$ & \\
\hline Insulation & Mineral wool & $\lambda$ & $0.038 \mathrm{~W} /(\mathrm{mK})$ & \\
\hline
\end{tabular}




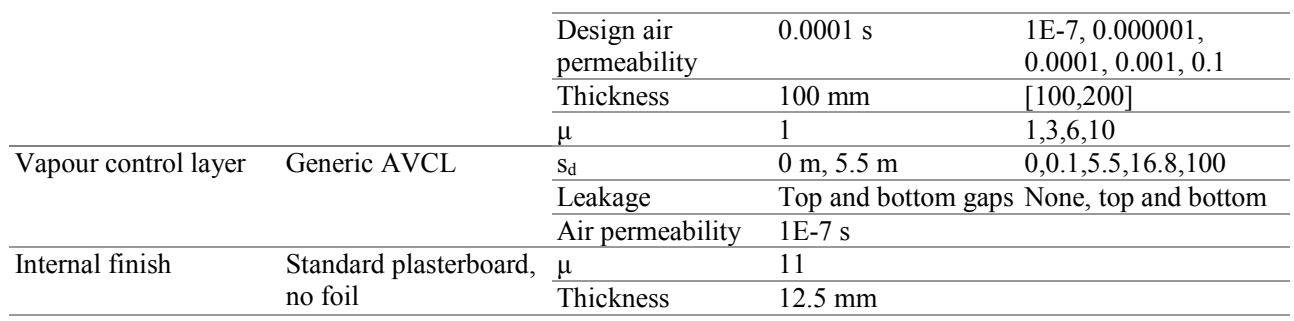

Table 2. Boundary conditions for parametric study.

\begin{tabular}{lll} 
Boundary conditions & Key parameters & Settings \\
\hline Indoor vapour pressure & Moisture load, according to BS EN 15026 [5] & Normal \\
\hline Indoor air overpressure & & $0 \mathrm{~Pa}$ \\
\hline Air flow behind cladding & Convective heat transfer coefficient $[4]$ & $8 \mathrm{~W} /\left(\mathrm{m}^{2} \mathrm{~K}\right)$ \\
\hline Outdoor climate & & Test Reference Year for \\
& & Heathrow (UK) - hourly data \\
\hline
\end{tabular}

\subsection{Failure criterion}

Moisture can accumulate within the wall structure, at the internal surface of the sheathing board. High moisture levels can lead to mould growth and wood rot of the board. Mould growth can occur on a surface if favourable conditions of temperature and relative humidity are maintained for a certain period of time. As mould growth occurs earlier than wood rot, the presence of mould at the surface of the sheathing board was used as failure criterion in this analysis.

The risk of mould growth within the wall was evaluated according to the criterion on surface water activity at critical area [6]. This criterion considers the influence of humidity and time of exposure on mould growth. Humidity is described by surface water activity, which is equivalent to equilibrium relative humidity. The moving average of relative humidity for a day, week and month is calculated and compared against the thresholds, shown in Table 3. Mould growth risk is represented in terms of percentage of instances above surface water activity threshold (i.e. the percentage of time each threshold is exceeded within a year).

Table 3. Thresholds for surface water activity criterion, adapted from Approved Document F [6].

Moving average period Surface water activity threshold Equilibrium RH threshold

\begin{tabular}{lll}
\hline 1 month & 0.75 & $75 \%$ \\
\hline $\mathbf{1}$ week & 0.85 & $85 \%$ \\
\hline $\mathbf{1}$ day & 0.95 & $95 \%$ \\
\hline
\end{tabular}

\section{Results}

Results showed that for the given structure and climate an AVCL between the plasterboard and the insulation is required. Therefore, the influence of vapour resistance on AVCL and of the presence of top and bottom air gaps was further studied.

\subsection{Influence of vapour resistance of AVCL and presence of air gaps}

Fig. 1 (left) shows mould growth risk for various levels of vapour resistance on the internal side of the insulation, considering top and bottom air gaps. Mould growth risk is high for all cases, but there is a reduction at vapour resistances associated with an equivalent air layer thickness $\left(\mathrm{s}_{\mathrm{d}}\right)$ of $5.5 \mathrm{~m}$; increasing the vapour resistance further from $\mathrm{s}_{\mathrm{d}}=5.5 \mathrm{~m}$ does not result in further improvement. 
As shown in Fig. 1 (right), in case of a VCL with medium to high vapour resistance, the risk is reduced if the AVCL is perfectly sealed (i.e. no air gaps), showing a clear influence of air gaps on mould growth risk. In case of low vapour resistance (e.g. $\left.\mathrm{s}_{\mathrm{d}}=0.1 \mathrm{~m}\right)$, the risk is high for both cases with and without air gaps.
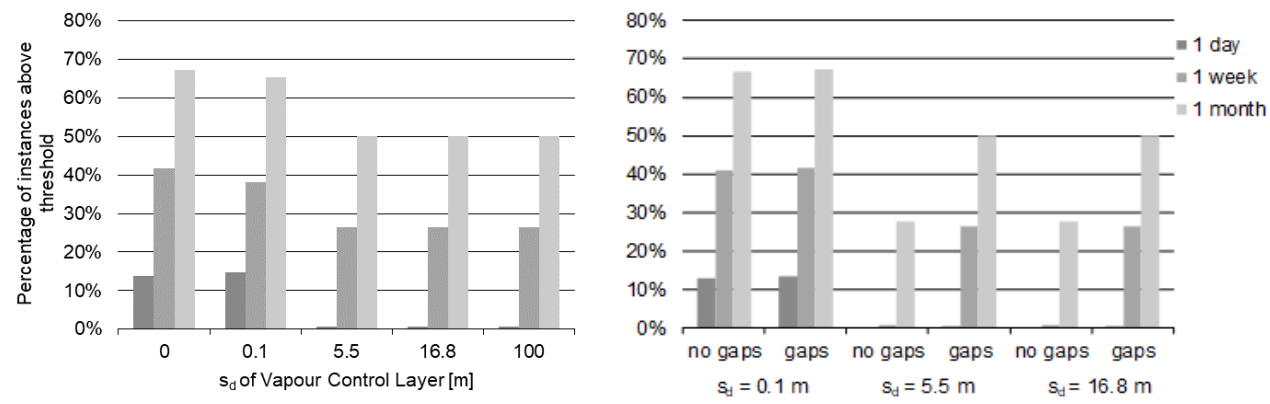

Fig. 1. Comparison of vapour control layers of different vapour resistance, denoted with the equivalent air layer thickness, $s_{d}[\mathrm{~m}]$ (left); comparison of cases with and without air gaps at the top and bottom of a wall, for two different vapour permeability values of the VCL (right).

\subsection{Influence of air and vapour permeability and thickness of mineral wool}

The influence of the air permeability of the mineral wool insulation layer was assessed assuming air gaps and a very airtight AVCL with no vapour resistance. The default air permeability of mineral wool was considered as $0.0001 \mathrm{~s}$, as defined in Delphin material database, where the range considered spans from airtight insulation (i.e. 1E-7 s) to an air permeability equivalent to the one of air (i.e. $0.1 \mathrm{~s}$ ). It is noted that these extreme values for the air permeability range are unlikely to represent mineral wool and are included for reference only. Fig. 2 (left) shows that decreasing the air permeability increases the mould growth risk at the interface between OSB and insulation.

The influence of the vapour permeability of mineral wool on mould growth risk (Fig. 2, right) was assessed, considering an airtight AVCL with gaps but with no vapour resistance. Results show a decreased risk of mould growth if the vapour permeability is reduced tenfold.
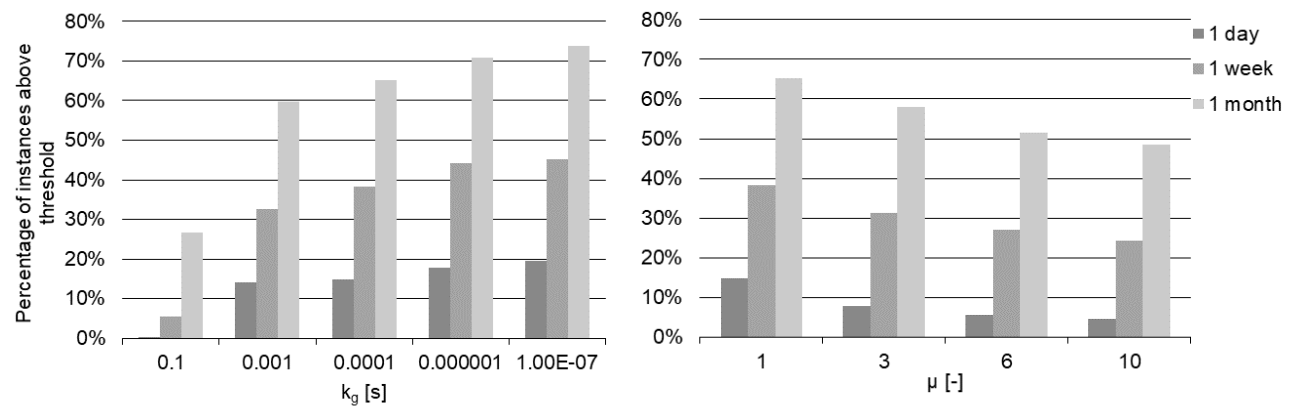

Fig. 2. Comparison of air permeability (left) and vapour permeability (right) for mineral wool.

The influence of the thickness of mineral wool was assessed considering 100, 150 and 200 $\mathrm{mm}$ for the insulation batt. This analysis considered an airtight AVCL with gaps and a medium vapour resistance (i.e. $\mathrm{s}_{\mathrm{d}}=5.5 \mathrm{~m}$ ). Fig. 3 (left) shows that the risk was found to be similar for the three cases, although slightly lower for increasing thicknesses. 


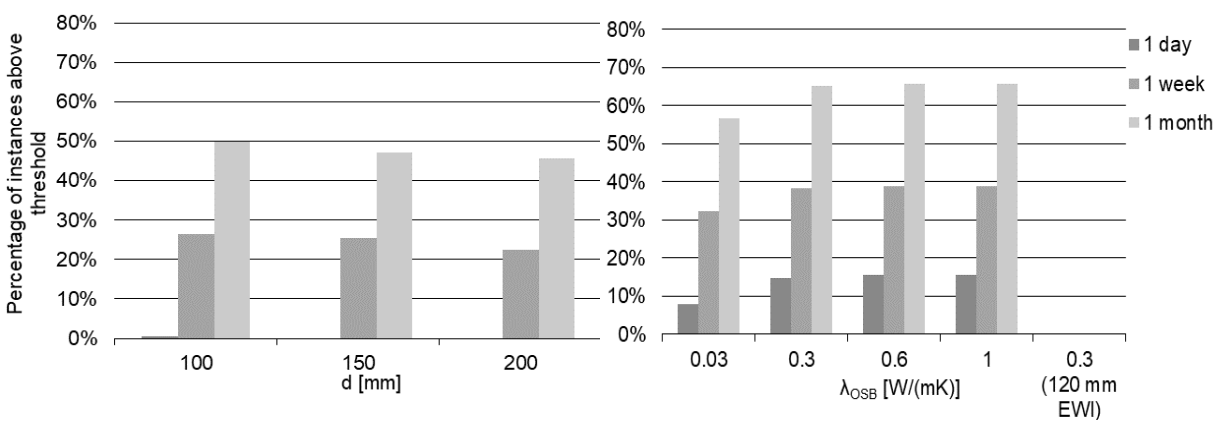

Fig. 3. Comparison of mineral wool thickness (left), considering a medium vapour resistance of the AVCL $\left(\mathrm{s}_{\mathrm{d}}=5.5 \mathrm{~m}\right)$. Comparison of thermal conductivity of OSB (right), assuming an airtight AVCL with gaps but with no vapour resistance; the comparison also shows a case with $120 \mathrm{~mm}$ of External Wall Insulation $(\lambda=0.035 \mathrm{~W} /(\mathrm{mK}))$, added to the outside of the external airtightness membrane.

\subsection{Influence of vapour permeability and thermal conductivity of OSB}

The influence of vapour permeability of the OSB was assessed assuming an airtight AVCL with gaps but with no vapour resistance. A negligible effect for moisture risk was found within the range of vapour permeability considered.

The influence of thermal conductivity of the OSB was also assessed. Within the thermal conductivity range identified for the OSB, there is not much difference between the moisture risk associated with a thermal conductivity of $0.3 \mathrm{~W} /(\mathrm{mK})$ and $1 \mathrm{~W} /(\mathrm{mK})$, which could be representative of OSB. As shown in Fig. 3 (right), with a thermal conductivity reduced to $0.03 \mathrm{~W} /(\mathrm{mK})$ - similar to insulation systems - the moisture risk was slightly reduced. To further analyse the influence of high external thermal resistance, an additional simulation was performed considering $120 \mathrm{~mm}$ of insulation installed on the outside of the analysed construction (see Fig. 3 (right)); this is the only solution that did not exceed the mould growth risk thresholds.

\section{Discussion}

The system analysed, a common type of timber frame wall in the UK, showed mould growth risk at all cases studied, even considering an internal AVCL. Vapour accumulates on the internal surface of the OSB, which is at low temperatures - hence high relative humidity - that may allow mould growth.

The results of the parametric study show that an AVCL with medium vapour resistance (e.g. $\mathrm{s}_{\mathrm{d}}=5.5 \mathrm{~m}$ ) can lower mould growth risk but did not remove it altogether; moreover, higher vapour resistances do not result in further risk reduction.

Also, results show that air gaps are worse than a perfect seal. In practice, a perfect seal is rarely achieved. Therefore, if one is to accept air gaps, there should be risk mitigation in place in other elements of the wall system.

The results suggest that, for the considered wall construction in temperate maritime climates, many moisture mechanisms interact but no moisture load is dominating. Results suggest that higher air permeability of insulation leads to reduced mould growth risk. Simulations showed that higher air permeability can cause warmer air to reach the critical area, as well as more vapour. However, the temperature increase due to the advection through air-permeable insulation might improve the hygric performance, probably at the expense of the thermal performance. These findings disagree with other studies, which found that cellulose was better than mineral wool because of lower air permeability, among 
various factors (e.g. moisture buffering) [7]. Alternatively, mould growth risk could be lowered by specifying materials that are less susceptible to mould growth, such as cement fibreboard.

The results of the parametric study showed very limited influence of insulation thickness. Moreover, thermal conductivity and vapour diffusion of OSB showed little or no influence in moisture risk; other types of sheathing boards, with a wider range of thermal resistance and vapour diffusion could be considered for further work. Furthermore, adding insulation to the outside of the sheathing board is effective for the reduction of mould growth risk.

\section{Conclusions}

This paper presented a parametric study to identify the mould growth risk associated to material properties, when external airtightness membranes are installed on a timber frame wall. The wall build-up considered was made of (outside to inside) an external airtightness membrane, OSB as sheathing board, mineral wool as insulation, AVCL and plasterboard, and the analysis was performed for a temperate maritime climate.

The system analysed showed high mould growth risk in the studied cases, although this is lowered in presence of an AVCL with medium-high vapour resistance, especially if sealed properly. Changing the thermal conductivity and vapour diffusion of OSB and the thickness of mineral wool did not lead to a reduced risk, whereas adding an insulation material with high thermal resistance to the outside of the wall can significantly reduce risk, suggesting that framed walls with external wall insulation are safer than common framed walls with insulation between studs only. Mould growth risk could be lowered by specifying different materials altogether for the sheathing board or the insulation; further work will consider this aspect.

The parametric study only considered timber cladding; however, brick cladding could lead to higher risk, because of lower ventilation [8] and higher risk of solar-driven vapour diffusion [9-11]. Finally, the study neglected As Built, In Service (ABIS) conditions associated with the external rainwater protection layer, such as defects to the flashing details or conditions associated with high initial moisture. These aspects should be considered in future work.

The authors would like to acknowledge the support of A Proctor Group.

\section{References}

[1] H. L. S. C. Hens, Build. Environ., vol. 91, pp. 138-151, 2015.

[2] L. Harriman, G. Brundrett, and R. Kittler, Humidity Control Design Guide for Commercial and Institutional Buildings. ASHRAE, 2001.

[3] J. Langmans, A. Nicolai, R. Klein, and S. Roels, Build. Environ., vol. 58, pp. 208-218, Dec. 2012.

[4] BSI, BS EN ISO 6946:2007.

[5] BSI, BS EN 15026:2007.

[6] HM Government, Approved Document F - Ventilation.HM Government, 2010.

[7] J. Langmans and S. Roels, J. Build. Perform. Simul., pp. 1-14, May 2014.

[8] M. Vanpachtenbeke, J. Langmans, J. Van den Bulcke, J. Van Acker, and S. Roels, Build. Environ., 2017.

[9] H. S. L. C. Hens, ASHRAE Trans., pp. 1077-1085, 2012.

[10]J. Lstiburek, "Solar Driven Moisture in Brick Veneer," 2008.

[11]A. Karagiozis, D. Derome, and J. Carmeliet, in 12th Canadian Conference on Building Science and Technology, 2009, pp. 85-96. 